Z. klin. Chem. u. klin. Biochem.

9. Jg., S. 489-490, November 1971

\title{
Elektronisch gesteuerte endogastrale Magensafttitration mit dem Intestinalsender
}

\author{
Von P. H. Wolters, M. Eckert und V. KLINGMÜlLER \\ Aus dem Klinisch-Chemischen Institut des Klinikums Manibeim, \\ II. Medizinische Fakultät der Universität Heidelberg
}

(Eingegangen am 13. Juli 1971)

Es wird eine Methode beschrieben, bei der die Vorteile des Intestinalsenders beibehalten werden und zusätzlich neben den bisher nur qualitativen und semiquantitativen jetzt auch quantitative Bestimmungen der Magenacidität möglich sind.

Dabei wird der Intestinalsender an einem etwa $1 \mathrm{~mm}$ dünnen, leicht beweglichen Schlauch angenäht, der von einer Bürette mit $1 \mathrm{M}$ $\mathrm{KHCO}_{3}$-Lösung über eine Schlauchpumpe in den Magen führt. An dem Empfänger des Intestinalsenders ist ein elektronischer Grenzwertschalter (Scrmmrt-Trigger) angeschlossen, der in einem pH-Bereich unter 3,5 die Schlauchpumpe einschaltet (Bicarbonat-Zufuhr) und über 3,5 ausschaltet. Auf diese Weise ist eine durch die sezernierte Magensäure selbst gesteucrte endogastrale Titration möglich.

\section{Electronically controlled endogastral titration of gastric juice with an intestinal transmitter}

A method is described in which the advantages of the intestinal transmitter are retained and, in addition to the qualitative and semiquantitative work that was hitherto possible, the new method permits the quantitative determination of stomach acidity.

The intestinal transmitter is sewn onto an easily movable tube of about $1 \mathrm{~mm}$ diameter, which leads into the stomach, via a peristaltic pump, from a burette containing $1 \mathrm{M} \cdot \mathrm{KHCO}_{3}$. The receiver of the intestinal transmitter is linked to a ScHMrrt trigger, which switches on the peristaltic pump (delivery of bicarbonate) when the $\mathrm{pH}$ falls below 3.5 and switches it off above this value. Thus the secretion of gastric juice controls its own titration.

Die Einführung des Intestinalsenders in die Magenfunktionsdiagnostik brachte gegenüber der Magensaftuntersuchung mit Schlauchsondierung eine fortschrittlichere und für den Patienten angenehmere Mèthode $(1,2)$. Abgesehen davon, daß die Schlauchsondierung für viele Patienten eine erhebliche physische und psychische Belastung darstellt, muß man bei dieser Methode auch mit Ergebnisverfälschungen rechnen $(3,4)$.

Einmal kommt es bei der Einführung des Schlauches durch Reizung sympatischer Fasern zu einer Verminderung der Magensaftsekretion, zum anderen bewirkt der Dauersog bei der Gewinnung das Magensaftes Pylorusöffnungen, die Magensaftverluste und Duodenalrefluxe zur Folge haben.

Diese Nebenerscheinungen sind bei der Untersuchung mit dem Intestinalsender bedeutend herabgesetzt, was die Einführung der Heidelberger Kapsel in die Magenfunktionsdiagnostik rechtfertigt. Allerdings muß die Methodik so gestaltet sein, daß sie hinsichtlich ihrer Aussagekraft und Reproduzierbarkeit anderen Untersuchungstechniken nicht nachsteht. Deshalb führten wir zur genauen quantitativen Enfassung der in der Zeiteinheit sezernierten aktuellen $\mathrm{H}^{+}=$Ionen eine neue $\mathrm{Me}-$ thode ein, die die Vorzüge des Intestinalsenders beibehält und außerdem die bisherigen Methoden an Genauigkeit übertrifft, weil die obengenannten Störfaktoren der Schlauchsondierung wegfallen.

\section{Beschreibung des Verfahrens}

Es handelt sich um eine durch die Magensäure selbst elektronisch gesteuerte endogastrale Titration (Abb.1). Bei dieser Methode wird der Intestinalsender an einem nur $1 \mathrm{~mm}$ dünnen Schlauch angenäht, der von einer 50-ml-Bürette mit $1 \mathrm{M} \quad \mathrm{KHCO}_{3}$-Lösung durch eine Schlauchpumpe zum Magen führt. Als Pumpe eignen sich die meisten Modelle der handelsüblichen Schlauch-

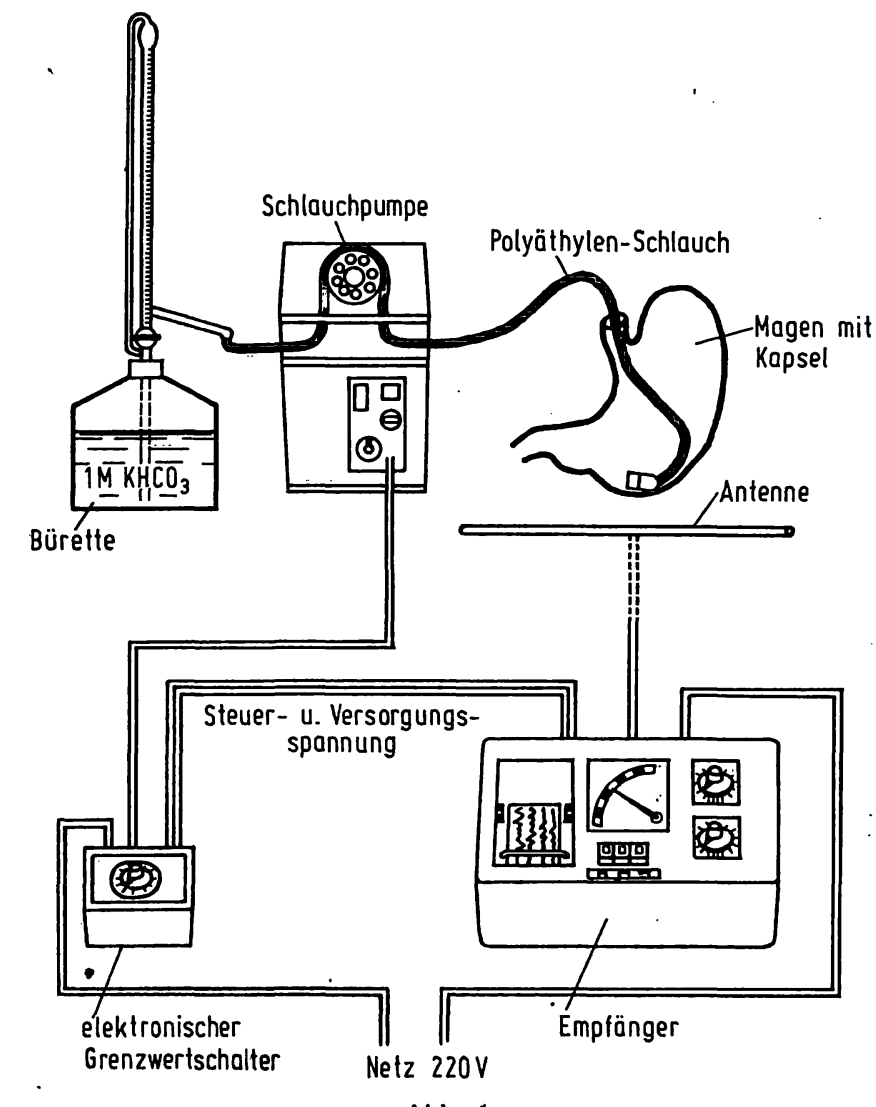

Abb. 1

Schematische Darstellung der Versuchsanordnung zur elektronisch gesteucrten endogastralen Titration. (Deutsclies Bundes-Pat. Nr. 
und Peristaltikpumpen. Die Kapsel selbst wird dabei an das Ende des Schlauches angenäht und das zu verschluckende Schlauchstück bei einer Länge von 45 bis $50 \mathrm{~cm}$ markiert. Das Verschlucken dieser Kạpel ist für den Patienten so leicht wie das Schlucken einer Kapsel an einem Catgutfaden, und der dünne bewegliche Schlauch hat keinerlei Reizwirkung. Wie üblich wird mit dem verschluckten Intestinalsender, der Antenne und dem Empfänger das $\mathrm{pH}$ gemessen. An dem $\mathrm{pH}-$ Meter des Empfängers ist ein Grenzwertschalter angeschlossen, der unter $\mathrm{pH} 3,5$ eingeschaltet und über $\mathrm{pH}$ 3,5 ausgeschaltet ist. Dieser Grenzwertschalter betätigt die Schlauchpumpe, so daß im sauren Bereich $\mathrm{KHCO}_{3}$ in den Magen hineingepumpt wird, bis $\mathrm{pH}$ 3,5 erreicht wird. $\mathrm{Ab} \mathrm{pH} 3,5$ und darüber bleibt die Pumpe so lange stehen, bis neu gebildete Magen- $\mathrm{HCl}$ den $\mathrm{pH}$-Wert wieder unter 3,5 senkt und dadurch die Pumpe wieder einschaltet.

Der Grenzwertschalter bestand in der ersten Entwicklungsstufe aus einem Zweitschreiber, der an den Schreiber des Empfängers angeglichen war. In diesem war ein mechanischer Kippschalter eingebaut, der bei pH 3,5 umschaltete (5). In neuester Zeit verwenden wir einen einfacheren elektronischen Grenzwertschalter, der ohne großen Kostenaufwand hergestellt und sehr leicht an den Empfänger angeschlossen werden kann. Es handelt sich um eine Schmirt-Trigger-Schaltung mit einem Magnetrelais, 3 Transistoren, 2 Dioden, 4 Widerständen und einem Potentiometer (Abb. 2).

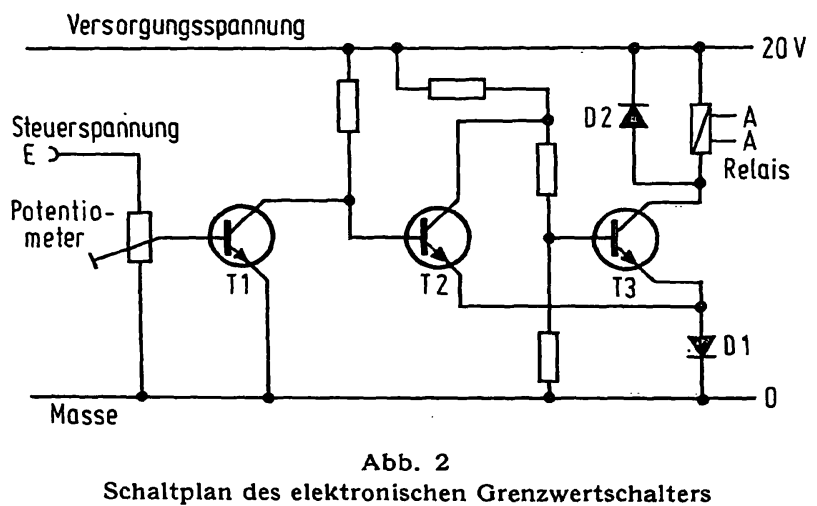

Den Grenzwert von pH 3,5 wählten wir deshalb, weil die Titration von $\mathrm{HCl}$ und $\mathrm{KHCO}_{3}$ einen Umschlagspunkt von $\mathrm{pH} 3,5$ hat und damit die vollständig dissoziierte $\mathrm{HCl}$ total erfaßt ist, während schwache Säuren (gebundene Säuren) bei diesem $\mathrm{pH}$ nicht dissoziiert sind und damit nicht erfaßt werden.

Die Justierung des Umschlagpunktes von $\mathrm{pH}$ 3,5 am Grenzwertschalter erfolgt nach der Aktivierung des Intestinalsenders und vor der Eichung des Empfängers, indem der Zeiger des pH-Meters mit Hilfe der Eichknöpfe auf $\mathrm{pH}$ 3,5 eingestellt und danach am Drehpotentiometer des Grenzwertschalters der Ansprechpunkt des Relais auf diesen Wert festgelegt wird.

Mit der hier aufgeführten Methode kann also über eine gewisse Zeit die Magen- $\mathrm{HCl}$ endogastral fortlaufend neutralisiert werden, so wie sie sezerniert wird, d. h. die in der Zeiteinheit sezernierten aktuellen $\mathrm{H}^{+}$-Ionen können direkt titriert werden. Die nach einer halben Stunde in der Bürette fehlende $\mathrm{KHCO}_{3}-\mathrm{Menge}$ ist äquivalent der Menge der in dieser Zeit sezernierten aktuellen $\mathrm{H}^{+}-$Ionen. Auf diese Weise kann ohne Stimulation die Basalsekretion, mit maximaler Stimulation durch Daueri. v.-Infusion von $6 \mu \mathrm{g}$ Pentagastrin/kg KG.Std. die maximal stimulierte Sekretion ermittelt werden. Wenn während dieser Untersuchung Magensaft durch den Pylorus entweicht, so ist das bei dieser Versuchsanordnung bedeutungslos, da dieser Magensaft ja bereits neutralisiert und damit erfaßt war.

\section{Bewertung des Verfahrens}

Die Genauigkeit der Methode wurde durch einen invitro-Modell-Versuch geprüft, in dem der Magen in dieser Versuchsanordnung durch einen Erlenmeyerkolben und die Belegzellen durch eine zweite Schlauchpumpe ersetzt wurden, die in 15 Einzelversuchen 2,0 bis $55,5 \mathrm{mMol} \mathrm{HCl} \mathrm{Std.} \mathrm{in} \mathrm{den} \mathrm{Erlenmeyerkolben} \mathrm{hinein-}$ pumpte. Die Beziehung der hineingepumpten 0,1N $\mathrm{HCl}-$ Menge zur titrierten $1 \mathrm{M} \mathrm{KHCO}_{3}$-Menge folgt einer Graden $(b=9,9893 ; a=0,9858 ; r=0,9989)$. Die endogastrale Titration erfolgt mit der gleichen Regelmäßigkeit der Umschaltungen des Grenzwertschalters und damit auch der Schlauchpumpe wie bei einem in-vitro-Versuch.

Die Höhe der Amplituden und die Frequenz der Umschaltungen ist von der Geschwindigkeit der Durchmischung der Magen-HCl mit $\mathrm{KHCO}_{3}$, d. h. von der Viskosität des Magensaftes und von der Magenperistaltik abhängig. Auch durch Lagewechsel und durch kräftige Palpation im epigastrischen Winkel konnte an diesem Verlauf nichts geändert werden. Also genügt die Magenperistaltik zum Durchmischen der Titrationsflüssigkeit mit der Magen- $\mathrm{HCl}$.

Neben der guten Reproduzierbarkeit zeigt die für den Patienten angenehme Methode weitere Vorteile. Die fortlaufende endogastrale Neutralisation vermeidet $\mathrm{Be}$ schwerden der durch maximale Stimulation bedingten Hypersekretion, hemmt Pylorusöffnungen und damit Doudenalrefluxe und setzt die Gefahr einer Ulcusperforation herab.

Im Vergleich zu derzeitig verwendeten, modernen Geräten zur Magensaftgewinnung liegen die Herstellungsund Beschaffungskosten für das hier beschriebene Gerät wesentlich niedriger. Außerdem benötigt diese Methode selbst für mehrere gleichzeitig laufende Untersuchungen nur eine Arbeitskraft.

\section{Literatur}

1. Ardenne, M. von und H. B. Sirung, Naturwissenschaften 45 , 564 (1958). - 2. Nöller, H. G., Fortschr. Med. 80, 351 (1962). 3. Schlotthauer, B. und H. G. Nöller, Münch. Med. Wschr. 17, 785 (1964). - 4. Pfister, U. H., R. Pirtkien und G. Seybold, Z. Gastroenterol. 7, 28 (1969). - 5. Wolters, P. H., M. ECKERT und V. KlingmüLler, Lesser-Loewe-Colloquium, Klinikum Mannheim (13. 1. 1971), ref. in Med. Tribune $\sigma$ (6), 1 und 28 (1971).

Dr. P. H. Wolters, M. Eckert, Prof. Dr. Dr. V. Klingmüller 68 Mannheim, Postfach 23 